\title{
Diagnosing Santa Ana Winds in Southern California with Synoptic-Scale Analysis
}

\author{
JOHN T. ABATZOGLOU AND RENAUd BARBERO \\ Department of Geography, University of Idaho, Moscow, Idaho \\ NiCHOLAS J. NAUSLAR \\ Division of Atmospheric Science, Desert Research Institute, Reno, Nevada
}

(Manuscript received 4 January 2013, in final form 6 March 2013)

\begin{abstract}
Santa Ana winds (SAW) are among the most notorious fire-weather conditions in the United States and are implicated in wildfire and wind hazards in Southern California. This study employs large-scale reanalysis data to diagnose SAW through synoptic-scale dynamic and thermodynamic factors using mean sea level pressure gradient and lower-tropospheric temperature advection, respectively. A two-parameter threshold model of these factors exhibits skill in identifying surface-based characteristics of SAW featuring strong offshore winds and extreme fire weather as viewed through the Fosberg fire weather index across Remote Automated Weather Stations in southwestern California. These results suggest that a strong northeastward gradient in mean sea level pressure aligned with strong cold-air advection in the lower troposphere provide a simple, yet effective, means of diagnosing SAW from synoptic-scale reanalysis. This objective method may be useful for medium- to extended-range forecasting when mesoscale model output may not be available, as well as being readily applied retrospectively to better understand connections between SAW and wildfires in Southern California.
\end{abstract}

\section{Introduction}

The Santa Ana winds (SAW) of southwestern California are characterized by strong offshore foehn winds that develop in the lee of the Transverse and Peninsular Ranges that are locally intensified by channeling through mountain gaps (e.g., Burrows 1987). SAW generally are a cool season phenomenon that develop after the dissipation of the North American monsoon as radiative cooling over the high plateau of the Great Basin gives way to a thermal continental high pressure. This thermal seasonal shift in conjunction with enhanced baroclinicity during the cool season favor the synoptic characteristics of SAW featuring a strong northeastward gradient in mean sea level pressure (MSLP) over southwestern California and northeasterly flow aloft in the wake of a frontal passage through the intermountain western United States (e.g., Small 1995; Raphael 2003). These conditions are manifested as mesoscale features through

Corresponding author address: Dr. John T. Abatzoglou, Dept. of Geography, University of Idaho, MS 3021, 875 Perimeter Dr., Moscow, ID 83844-3021.

E-mail: jabatzoglou@uidaho.edu gravity mountain waves in a stably stratified atmosphere with a strong cross-barrier potential temperature gradient. Consequently, there is a downward momentum flux that results in strong subsidence and an acceleration of gap winds leeward (west and south) of the Transverse and Peninsular Ranges with the strongest winds associated with the hydraulic jump phenomena in the wake of a topographic barrier (e.g., Gaberšek and Durran 2006; Durran 1990; Hughes and Hall 2009). The combination of strong winds and extremely dry air juxtaposed with ample and available fuel is conducive to the rapid rate of spread of fire fronts and extreme wildfire behavior in the extensive wildland urban interface in the Los Angeles and San Diego basins and pose significant direct and indirect hazards to human infrastructure and health (e.g., Schroeder et al. 1964, 264-274; Westerling et al. 2004; Keeley et al. 2004).

Numerous efforts have been made to qualify SAW using weather observations from individual stations (e.g., Edinger et al. 1964), synoptic pattern recognition (e.g., Schroeder et al. 1964, 264-274; Raphael 2003), and mesoscale and synoptic-scale reanalysis (e.g., Hughes and Hall 2009; Jones et al. 2010). Each method has its inherent strengths and weaknesses, as well as prognostic 
and diagnostic utility; however, an accepted definition of a SAW is lacking. Operationally, the National Weather Service Forecast Office of Los Angeles/Oxnard (NWSFO LOX) broadly characterizes SAW as an "offshore flow from the east or northeast of 25 knots $\left(12.8 \mathrm{~m} \mathrm{~s}^{-1}\right)$ or greater associated with very low relative humidity." However, this definition fails to specify the temporal or spatial characteristics and SAW are subjectively classified during the months of September-April (NWSFO LOX 2007, personal communications). Small (1995) notes a SAW criterion of at least a 10-hPa MSLP difference between Los Angeles, California (KLAX), and Tonopah, Nevada (KTPH), and further classifies events into those purely driven by MSLP gradient versus "stronger" events with upper-level support that reinforce subsidence and allow for downward momentum flux. More recently, Hughes and Hall (2009) identified SAW from mesoscale model output using a threshold for offshore surface wind speed over a small area of the Santa Monica Mountains and offshore waters, whereas Jones et al. (2010) identified SAW using MSLP fields from coarsescale reanalysis. Unfortunately, these diagnostics either require mesoscale model output or are weakly defined and, moreover, have not been validated by surface-based observations.

We build upon previous studies to develop an objective method for qualifying SAW using diagnostics that represent key dynamical and thermodynamical processes. We hypothesize that SAW can be efficiently identified using synoptic-scale diagnostics that feature offshore surface gradient flow phase locked with lowertropospheric winds and subsidence forced via cold-air advection (CAA). This procedure is not intended to supplant mesoscale models but, rather, to complement them in medium-range operational forecasting, as well as to better understand the role of SAW on the fire history of Southern California.

\section{Data and methods}

The majority of previous diagnostics of SAW have used station data and synoptic analyses (e.g., McCutchan and Schroeder 1973; Schroeder et al. 1964, 264-274; Small 1995). Observations can provide invaluable local data; however, local observations may be problematic as a direct diagnostic due to the fact that they are generally temporally limited, and local observations of surface wind speed, temperature, and humidity may be biased by topographic complexity and not be reflective of the broader region. By contrast, mesoscale models have been shown to resolve the spatiotemporal details of SAW and are used operationally for short-range forecasting (e.g., Conil and Hall 2006; Hughes and Hall 2009). While the observational network and mesoscale models are essential for capturing the spatial structure and intensity of SAW, the present study focuses on developing and evaluating diagnostics using coarse-scale reanalysis that captures the synoptic-scale signatures of SAW, rather than the local details of SAW. The advantages of reanalysis datasets include spatial and temporal completeness of diagnostic fields spanning numerous decades as well as potential transferability to both operational medium- and extended-range forecasting and global climate modeling experiments.

Building on previous studies that have used both synoptic (e.g., Schroeder et al. 1964, 264-274; Raphael 2003; Jones et al. 2010) and mesoscale (Hughes and Hall 2009) diagnostics to identify SAW, we hypothesize that two key atmospheric diagnostics effectively synthesize ingredients conducive to SAW events: 1) a strong northeastward pressure gradient across southwestern California and 2) strong CAA over the deserts of Southern California inland of the Transverse Range. The former criterion has been applied both operationally and in research studies (NWSFO LOX 2007, personal communication; Miller and Schlegel 2006; Jones et al. 2010), and Small (1995) showed that a KTPH-KLAX gradient of $10 \mathrm{hPa}$ is a typical SAW threshold. Schroeder et al. (1964, 264-274) and Small (1995) alluded to the fact that the latter criterion was important in supporting intense events, notably in the wake of a trough passing north and east of southwestern California. Hughes and Hall (2009) showed that the strength of the lower-tropospheric temperature gradient (approximately $850 \mathrm{hPa}$ ) between the interior deserts of Southern California and offshore is the dominant control on the strength of SAW and facilitates local leeside intensification of winds. Cold-air advection and the associated subsidence when directionally aligned with surface-based winds strengthen downward momentum flux and accelerate lower-level winds particularly in the lee of a mountain barrier (e.g., Gaberšek and Durran 2006). Unlike the MSLP gradient criterion, no established criterion has been examined for CAA.

Both MSLP gradient and 850-hPa temperature advection are readily computed from daily National Centers for Environmental Prediction -National Center for Atmospheric Research (NCEP-NCAR) reanalysis data from 1948 to 2010. We calculate the MSLP gradient [hPa $(100 \mathrm{~km})^{-1}$ ] between KPTH and KLAX through bilinear interpolation (Fig. 1a). Temperature advection at $850 \mathrm{hPa}$ is considered over the domain of the interior deserts of Southern California (Schroeder et al. 1964, 264-274; Small 1995; inset box in Fig. 1a). Temperature advection is independent of MSLP gradient (e.g., CAA can occur without a strong onshore MSLP gradient); however, the presence of both strong 
(a) RAWS 1996-2010

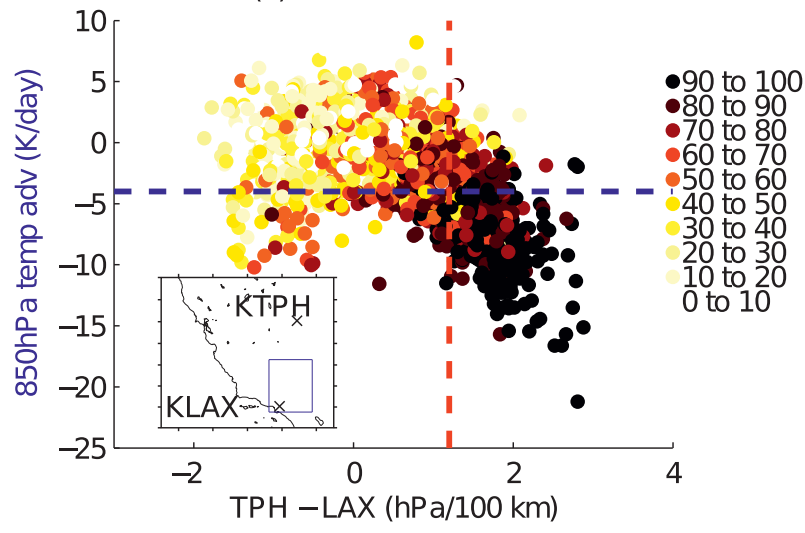

(b) Daily mean wind \& FFWI in surface (RAWS)

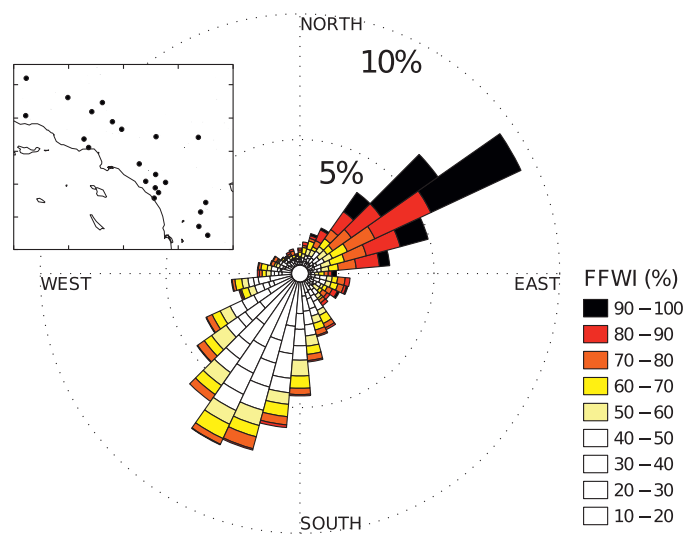

FIG. 1. (a) Daily MSLP gradient $\left[\mathrm{hPa}(100 \mathrm{~km})^{-1}\right]$ between KTPH and KLAX vs 850-hPa temperature advection over southern CA (denoted in inset) for Sep-Apr 1948-2010 calculated from daily NCEP-NCAR reanalysis. The color of each circle indicates the mean FFWI calculated from hourly RAWS observations from 22 RAWS across southern CA (denoted in inset) during 1996-2010. Thresholds of $1.2 \mathrm{hPa}(100 \mathrm{~km})^{-1}$ and $-4 \mathrm{~K} \mathrm{day}^{-1}$ are chosen to delineate SAW cutoffs. (b) Wind rose of daily mean wind direction and the related daily mean FFWI expressed in percentiles from the 22 RAWS for Sep-Apr 1996-2010.

CAA and an onshore MSLP gradient would have lowertropospheric flow directionally in phase with surfacebased winds to further downward mass fluxes and leeside winds.

Hourly surface observations from 22 Remote Automated Weather Stations (RAWS) located in areas prone to extreme fire weather are used for validation (Fig. 1b). These 22 stations are selected from a larger suite of RAWS in southwestern California because of data completeness $(<10 \%$ of hourly observations missing) from 1996 to 2010 . We qualify a SAW day when 1) the daily mean (computed for the previous $24 \mathrm{~h}$ ending at 0000 UTC for consistency with the reanalysis)
Fosberg fire weather index (FFWI; Fosberg 1978) exceeds the 90th percentile for September-April (Sep-Apr) and 2) the mean standardized wind direction is between north-northwest $\left(330^{\circ}\right)$ and clockwise to south-southeast $\left(120^{\circ}\right)$. A SAW day is classified as an extreme SAW day when the daily mean FFWI exceeds the 97th percentile. The FFWI is computed for each station on an hourly basis using relative humidity, temperature, and wind speed. The FFWI is a fire danger metric designed to track fine fuel moistures and short-term fire weather irrespective of antecedent fuel moistures, yet has been shown to be associated with large autumn fires in southwestern California (e.g., Moritz et al. 2010). For this reason, FFWI may be somewhat biased toward warmer SAW events important for wildfire hazards and less optimal for midwinter SAW events that occur under cooler conditions. Daily mean wind direction is calculated by normalizing hourly wind speed for each station by dividing by the Sep-Apr mean wind speed and calculating an average of zonal and meridional hourly wind velocities across the 22 stations. This step is taken to avoid biases imparted by particularly windy stations in the overall mean wind direction.

Relationships between the two diagnostics and the occurrence of both SAW and extreme SAW days are examined qualitatively. From these qualitative observations we explore both a two-parameter threshold exceedance model that includes MSLP and 850-hPa temperature advection, as well as one-parameter threshold exceedance models that only include one diagnostic. Model skill is evaluated using the Heidke skill score (HSS; Wilks 2006) for combinations of MSLP gradient every $0.02 \mathrm{hPa}$ between 0 and $2 \mathrm{hPa}(100 \mathrm{~km})^{-1}$ and $850-\mathrm{hPa}$ temperature advection every $0.1 \mathrm{~K} \mathrm{day}^{-1}$ between 0 and $-10 \mathrm{~K} \mathrm{day}^{-1}$. We extract the highest HSS from the matrix of MSLP gradient and temperature advection thresholds and compare the two-parameter approach to one that only uses MSLP gradient or 850-hPa temperature advection.

\section{Results}

Figure 1a shows a scatterplot of daily MSLP gradient [expressed in $\mathrm{hPa}(100 \mathrm{~km})^{-1}$ ] and temperature advection at $850 \mathrm{hPa}$ and the associated daily mean FFWI computed from surface stations data. Days with high ( $>90$ th percentile) and extreme ( $>97$ th percentile) FFWI occur almost exclusively on days with prevailing north to easterly surface winds, with only $0.6 \%(0 \%)$ of days with high (extreme) fire danger not having prevailing northeasterly surface winds characteristic of SAW (Fig. 1b). Figure 1a illustrates the proclivity of high FFWI days coincident with strong CAA and a large KTPH-KLAX 


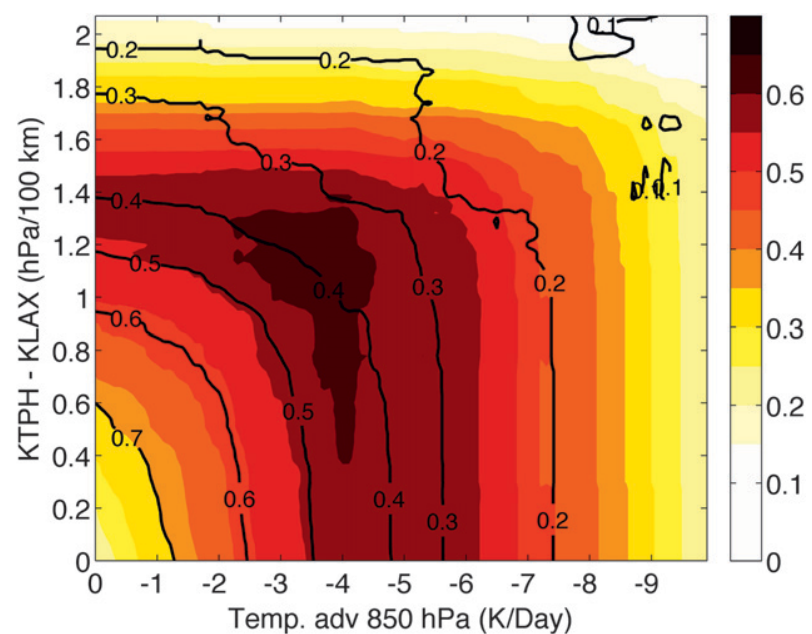

FIG. 2. HSS (shaded) and false alarm ratio (FAR, contoured) between SAW observed from RAWS and SAW estimated from the NCEP-NCAR MSLP gradient and 850-hPa temperature advection (1996-2010). Scores are computed iteratively for all combinations every $0.1 \mathrm{~K}$ day $^{-1}$ between 0 and $-10 \mathrm{~K}$ day $^{-1}$ (temperature advection) and every $0.02 \mathrm{hPa}$ between 0 and $2 \mathrm{hPa}(100 \mathrm{~km})^{-1}$ (MSLP gradient).

MSLP gradient. Approximately $76 \%$ and $94 \%$ of all SAW and extreme SAW days occur with daily MSLP gradients $>1.2 \mathrm{hPa}(100 \mathrm{~km})^{-1}$ and $850-\mathrm{hPa}$ temperature advection $<-4 \mathrm{~K} \mathrm{day}^{-1}$, respectively. Moreover, extreme and high FFWI values are rarely identified outside these bounds and are far less prevalent when there is strong CAA in the absence of a strong MSLP gradient or where there is a strong MSLP in the absence of strong CAA. Hence, although the parameters are significantly correlated $(r=-0.51)$, they represent complementary processes whereby their union is associated with the highest probability of SAW conditions.

Thresholds were objectively determined based on the sensitivity analysis of HSS using variations of the two parameters to identify surface-based SAW and extreme SAW $\left(\right.$ FFWI $>97$ th percentile and winds between $330^{\circ}$ and $120^{\circ}$ ) over the common period (1996-2010). The highest HSS for SAW is found with a threshold of MSLP gradient of $1.2 \mathrm{hPa}(100 \mathrm{~km})^{-1}$ and a threshold of temperature advection of $-4 \mathrm{~K} \mathrm{day}^{-1}$ (Fig. 2). These thresholds correspond to the 85th percentile of Sep-Apr values for both MSLP gradient and CAA. By comparison, the $1.2 \mathrm{hPa}(100 \mathrm{~km})^{-1}$ MSLP gradient from the reanalysis is approximately the 83rd percentile of the daily averaged KTPH-KLAX MSLP gradient, thereby demonstrating a close correspondence between reanalysis and surface-based MSLP thresholds. A similar analysis using only extreme SAW days exhibits a similar finding, albeit with stronger thresholds of approximately $1.6 \mathrm{hPa}(100 \mathrm{~km})^{-1}(95$ th percentile $)$ and approximately
TABLE 1. Skill scores between SAW observed from RAWS (FFWI $>90$ th percentile and winds between $330^{\circ}$ and $120^{\circ}$ ) and SAW estimated from MSLP [threshold fixed at $1.2 \mathrm{hPa}(100 \mathrm{~km})^{-1}$ ], temperature advection at $850 \mathrm{hPa}$ (threshold fixed at $-4 \mathrm{~K}^{\text {day }}{ }^{-1}$ ), and both variables during the 1996-2010 period. Boldface values indicate the best combination for each score.

\begin{tabular}{lccc}
\hline \hline & MSLP & $\begin{array}{c}\text { Temperature } \\
\text { advection }\end{array}$ & $\begin{array}{c}\text { MSLP + temperature } \\
\text { advection }\end{array}$ \\
\hline Hit rate & 0.78 & $\mathbf{0 . 8 4}$ & 0.71 \\
Percent correct & 0.89 & 0.86 & $\mathbf{0 . 9 3}$ \\
FAR & 0.52 & 0.59 & $\mathbf{0 . 3 9}$ \\
Bias & 1.63 & 2.04 & $\mathbf{1 . 1 6}$ \\
Threat score & 0.42 & 0.38 & $\mathbf{0 . 4 9}$ \\
HSS & 0.53 & 0.47 & $\mathbf{0 . 6 2}$ \\
\hline
\end{tabular}

$-8 \mathrm{~K} \mathrm{day}^{-1}$ (96th percentile) for MSLP and temperature advection, respectively. These results elucidate the advantage of a two-parameter approach over that using only an MSLP gradient or $850-\mathrm{hPa}$ temperature advection. Tables 1 and 2 show skill scores using MSLP only, 850-hPa temperature advection only, and the twoparameter approach for SAW and extreme SAW. All measures of skill aside from hit rate are improved using the two-parameter threshold approach variables. For extreme SAW, the sensitivity analysis suggests that thermodynamic forcing supersedes pressure gradient forcing, confirming the results of Hughes and Hall (2009). The inclusion of additional synoptic-scale factors, including negative vorticity advection over the inland deserts of Southern California, did not yield significant improvements in model performance.

A composite of MSLP and 850-hPa winds and temperature advection coincident with SAW days is shown in Fig. 3a. Synoptic features characteristic of SAW in the literature (e.g., Raphael 2003) include a strong northnortheastward MSLP gradient and often a prominent high pressure region over the Great Basin. We note that SAW days can, however, occur without a strong high

TABLE 2. Skill scores between extreme SAW observed from RAWS (FFWI $>97$ th percentile and winds between $330^{\circ}$ and $120^{\circ}$ ) and SAW estimated from MSLP [threshold fixed at $1.6 \mathrm{hPa}$ $(100 \mathrm{~km})^{-1}$ ], temperature advection at $850 \mathrm{hPa}$ (threshold fixed at $-8 \mathrm{~K} \mathrm{day}^{-1}$ ), and both variables during the 1996-2010 period. Boldface values indicate the best combination for each score.

\begin{tabular}{lccc}
\hline \hline & MSLP & $\begin{array}{c}\text { Temperature } \\
\text { advection }\end{array}$ & $\begin{array}{c}\text { MSLP + temperature } \\
\text { advection }\end{array}$ \\
\hline Hit rate & $\mathbf{0 . 6 5}$ & 0.65 & 0.54 \\
Percent correct & 0.94 & 0.95 & $\mathbf{0 . 9 7}$ \\
FAR & 0.72 & 0.67 & $\mathbf{0 . 5 6}$ \\
Bias & 2.33 & 1.96 & $\mathbf{1 . 2 1}$ \\
Threat score & 0.24 & 0.28 & $\mathbf{0 . 3 2}$ \\
HSS & 0.36 & 0.42 & $\mathbf{0 . 4 6}$ \\
\hline
\end{tabular}


(a) SLP + CAA \& Winds @850 hPa during SAW

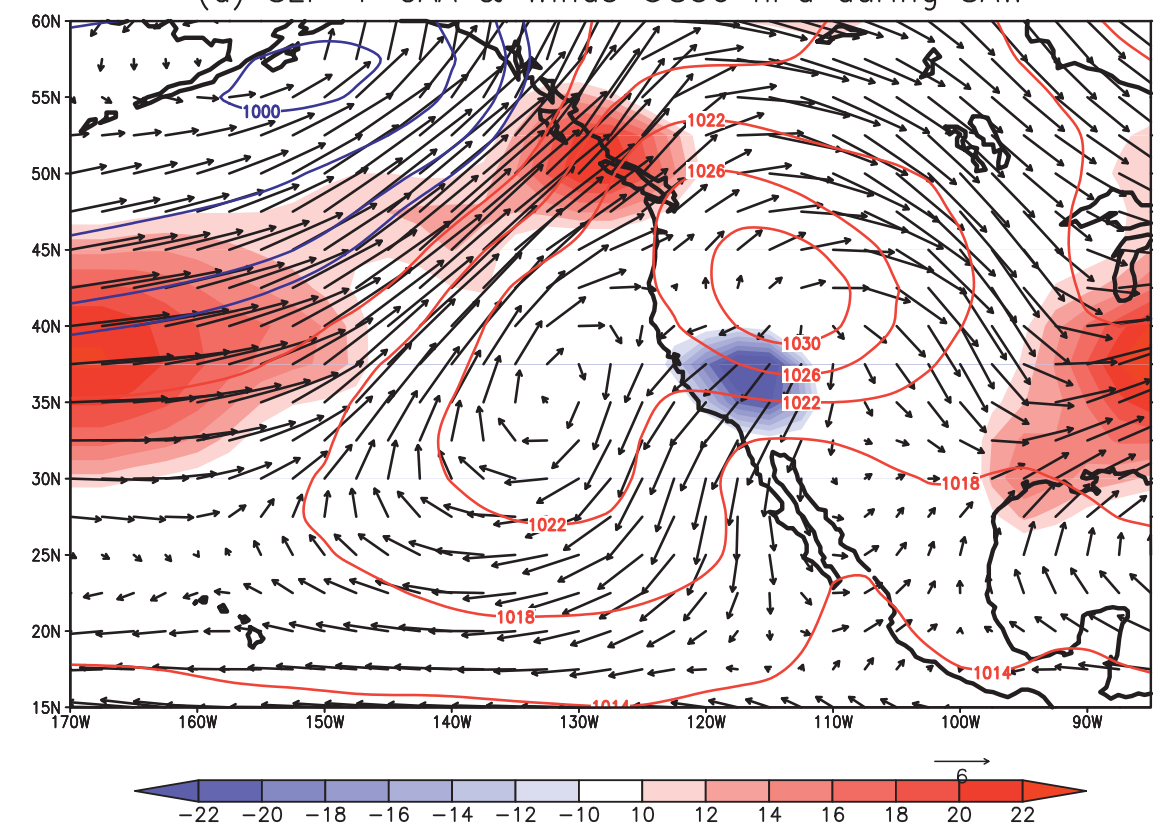

(b) FFWI (percentiles) during SAW

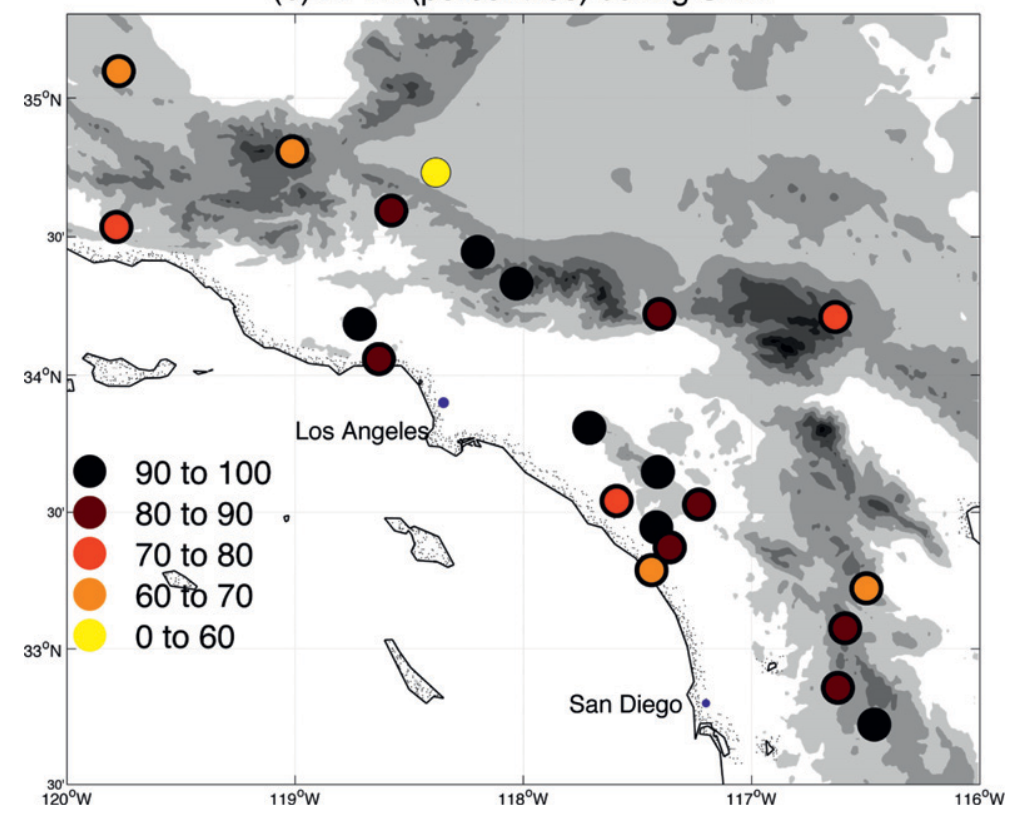

FIG. 3. (a) MSLP (contoured, hPa), mean temperature advection at $850 \mathrm{hPa}$ (shaded, $\mathrm{K}_{\text {day }}{ }^{-1}$ ), and $850-\mathrm{hPa}$ mean winds (vectors, $\mathrm{m} \mathrm{s}^{-1}$ ) for SAW defined from NCEP-NCAR variables (1948-2010). (b) Daily mean FFWI (\%) observed at RAWS during SAW days defined from NCEP-NCAR variables (1996-2010). Boldface circles indicate values significantly different from the daily mean state at the $95 \%$ level according to a $t$ test.

over the Great Basin with over half of SAW days having MSLP averaged over $37.5^{\circ}-42.5^{\circ} \mathrm{N}, 112.5^{\circ}-117.5^{\circ} \mathrm{W}$ less than $1030 \mathrm{hPa}$. Directional alignment of surface and lower-tropospheric flows with backing winds at higher levels (not shown) and CAA over the mountains and inland deserts of Southern California provide additional reinforcement for the downward momentum transport of winds. Negative vorticity advection at $500 \mathrm{hPa}$ (not shown) and associated quasigeostrophic subsidence are observed over the inland deserts of Southern California 
(a)

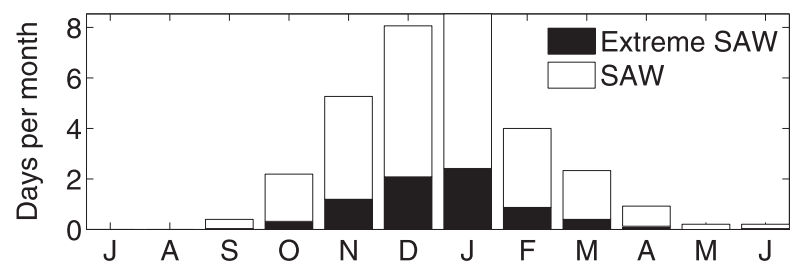

(b)

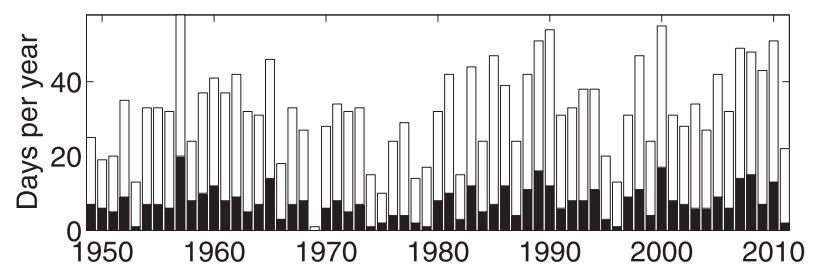

FIG. 4. (a) Monthly climatology and (b) interannual variability (Jul-Jun) of extreme (black) and all (white) SAW days (1948-2010).

into southern Nevada during SAW in the wake of trough passage northeast of the study area that further contribute to the synoptic-scale forcing. Figure $3 b$ shows the daily mean FFWI recorded by RAWS surface stations during SAW days (1996-2010). All stations (except for one located inland of the Transverse Range) exhibit statistically significant high fire danger, typically above the 85th percentile (Fig. 3b). For extreme SAW days, most leeward stations have an FFWI exceeding the 95th percentile (not shown). High fire danger reflected in FFWI during SAW days is ultimately traced back to anomalously low daily mean relative humidity $(<30 \%$, or nearly $30 \%$ lower than climatology) and daily mean wind speeds typically $50 \%$ higher than climatology.

Climatology of SAW and extreme SAW over the reanalysis record (1948-2010) is created based on the two-parameter threshold approach. These results mirror the seasonal nature of prior studies with a unimodal peak during the cool season (e.g., Raphael 2003; Jones et al. 2010) adhering to the mean seasonal cycle of the MSLP gradient and temperature advection with an average of 24.6 and 7.5 days $\mathrm{yr}^{-1}$ identified as SAW and extreme SAW days, respectively (Fig. 4a). The midwinter peak in SAW events (not shown, but similar to days) is more pronounced than has been found in previous studies (Raphael 2003), but consistent with Jones et al. (2010). The limited 15-yr climatology of surface station-based SAW showed relatively more days during early autumn and spring with FFWI exceeding the 90th percentile than our two-parameter model; however, the predisposition of FFWI to warmer temperatures and lower relative humidity likely contributes to this bias. Figure $4 \mathrm{~b}$ shows interannual variability of the number of SAW and extreme SAW events over the cool season
(July-June). A significant interannual correlation was found between the frequency of SAW $(r=0.84)$ and extreme SAW $(r=0.77)$ days between the two-parameter and station-based diagnostics.

The chronology of SAW using the two-parameter threshold model was also able to detect 1) all major SAW events as compiled by the San Diego and Oxnard NWSFO's from 1995 to 2007 (NWSFO LOX 2007, personal communications) and 2) $89 \%$ of dates corresponding to all wildfire and wind-related damage storm events in San Diego, Orange, Los Angeles, Ventura, Riverside, and San Bernardino Counties from 1 October 2006 to 31 December 2010 that contained the terminology "Santa Ana winds" as part of the event narrative (NCDC 2012).

\section{Conclusions}

Lower-tropospheric temperature advection and MSLP derived from coarse-scale reanalysis efficiently capture thermodynamic and dynamic processes that characterize SAW, as elaborated in prior studies. Threshold sensitivity analysis revealed thresholds of $1.2 \mathrm{hPa}(100 \mathrm{~km})^{-1}$ and $-4 \mathrm{~K} \mathrm{day}^{-1}$ for SAW, with more stringent temperature advection thresholds for extreme SAW. Independent observations from a RAWS mesonet verify the utility of this methodology in capturing days with critical fire weather and prevailing northeasterly winds that interact with the complex dynamics of terrain and fuels in the region to produce wildfire hazards (e.g., Sharples 2009).

Mesoscale models are the logical choice to provide superior information on SAW and their subsynopticscale manifestation over the complex terrain of southwestern California (e.g., Conil and Hall 2006; Huang et al. 2009); however, the empirical model established in this study is complementary and may be advantageous in medium-range and ensemble-based forecasts. In addition, the simplicity of the proposed methodology lends itself to usage in a research setting where more computationally intensive mesoscale models may not be readily available. For example, the retrospective chronology of SAW and extreme SAW may allow for a more thorough understanding of the role of SAW and the weather-climate continuum on the fire history of Southern California, as well as potential modulations in SAW with low-frequency climate variability and climate change (e.g., Keeley 2004; Miller and Schlegel 2006; Hughes et al. 2011).

Acknowledgments. The authors appreciate the constructive reviews by three anonymous reviewers. This research was supported by NSF Grant ATM-0801474, the NSF Idaho EPSCoR Program under Award EPS-0814387, 
and the Joint Fire Science Program under Awards 08-1-119 and 11-1-7-4. A chronology of SAW days identified using this approach is available online (at http://nimbus. cos.uidaho.edu/JFSP/pages/publications.html).

\section{REFERENCES}

Burrows, L. D., 1987: Development of forecast guidance for Santa Ana conditions. Natl. Wea. Dig., 12 (1), 4-11.

Conil, S., and A. Hall, 2006: Local regimes of atmospheric variability: A case study of Southern California. J. Climate, 19, 4308-4325.

Durran, D. R., 1990: Atmospheric Processes over Complex Terrain. Meteor. Monogr., No. 45, Amer. Meteor. Soc., 394 pp.

Edinger, J. G., R. A. Helvey, and D. Baumhefner, 1964: Surface wind patterns in the Los Angeles basin during "Santa Ana" conditions. USDA Forest Service Research Project 2606, Part 1, Los Angeles, CA, 84 pp.

Fosberg, M. A., 1978: Weather in wildland fire management: The fire weather index. Proc. Conf. on Sierra Nevada Meteorology, Lake Tahoe, CA, Amer. Meteor. Soc, 1-4.

Gaberšek, S., and D. Durran, 2006: The dynamics of gap flow over idealized topography. Part II: Effects of rotation and surface friction. J. Atmos. Sci., 63, 2720-2739.

Huang, C., Y.-L. Lin, M. L. Kaplan, and J. J. Charney, 2009: Synopticscale and mesoscale environments conducive to forest fires during the October 2003 extreme fire event in Southern California. J. Appl. Meteor. Climatol., 48, 553-559.

Hughes, M., and A. Hall, 2009: Local and synoptic mechanisms causing Southern California's Santa Ana winds. Climate Dyn., 34, 847-857, doi:10.1007/s00382-009-0650-4.

- — , and J. Kim, 2011: Human-induced changes in wind, temperature and relative humidity during Santa Ana events. Climatic Change, 109 (Suppl.), 119-132, doi:10.1007/ s10584-011-0300-9.
Jones, C., F. Fujioka, and L. M. V. Carvalho, 2010: Forecast skill of synoptic conditions associated with Santa Ana winds in Southern California. Mon. Wea. Rev., 138, 4528-4541.

Keeley, J. E., 2004: Impact of antecedent climate on fire regimes in coastal California. Int. J. Wildland Fire, 13, 173-182.

- C. J. Fotheringham, and M. A. Moritz, 2004: Lessons from the October 2003 wildfires in Southern California. J. For., 102, 26-31.

McCutchan, M. H., and M. J. Schroeder, 1973: Classification of meteorological patterns in Southern California by discriminant analysis. J. Appl. Meteor., 12, 571-577.

Miller, L. N., and N. J. Schlegel, 2006: Climate change projected fire weather sensitivity: California Santa Ana wind occurrence. Geophys. Res. Lett., 33, L15711, doi:10.1029/2006GL025808.

Moritz, M. A., T. J. Moody, M. A. Krawchuk, M. Hughes, and A. Hall, 2010: Spatial variation in extreme winds predicts large wildfire locations in chaparral ecosystems. Geophys. Res. Lett., 37, L04801, doi:10.1029/2009GL041735.

National Climatic Data Center, cited 2012: Storm events database. [Available online at www.ncdc.noaa.gov/stormevents/.]

Raphael, M. N., 2003: The Santa Ana winds of California. Earth Interact., 7. [Available online at http://EarthInteractions.org.]

Schroeder, M. J., M. Glovinsky, V. F. Henricks, F. C. Hood, and M. K. Hull, 1964: Synoptic Weather Types Associated with Critical Fire Weather. Pacific Southwest Forest and Range Experiment Station, Berkeley Press, 492 pp.

Sharples, J. J., 2009: An overview of mountain meteorological effects relevant to fire behaviour and bushfire risk. Int. J. Wildland Fire, 18, 737-754.

Small, I. J., 1995: Santa Ana winds and the fire outbreak of fall 1993. NOAA Tech. Memo. NWS WR-230, 48 pp.

Westerling, A. L., D. R. Cayan, T. J. Brown, B. L. Hall, and L. G. Riddle, 2004: Climate, Santa Ana winds and autumn wildfires in Southern California. Eos, Trans. Amer. Geophys. Union, 85, 289-296.

Wilks, D. S., 2006: Statistical Methods in the Atmospheric Sciences. 2nd ed. Academic Press, 648 pp. 